Artículos Científicos

\title{
Opinión de la comunidad de Huajintlán, Morelos, sobre la vinculación con estudiantes de nivel superior
}

\author{
Opinion of the Community of Huajintlán, Morelos, About Linkage with Higher \\ Education Students
}

\begin{abstract}
Opinião da comunidade de Huajintlán, Morelos, sobre a relação com os estudantes de nível superior
\end{abstract}

Erika Román Montes de Oca

Universidad Autónoma del Estado de Morelos, Morelos, México

erika.romanm@uaem.edu.mx

http://orcid.org/0000-0002-9490-6422

Jesús Eduardo Licea Resendiz

Universidad Autónoma del Estado de Morelos, Morelos, México

jesus.eduardo@uaem.mx https://orcid.org/0000-0002-4468-3435

José Luís García Hernández

Universidad Autónoma del Estado de Morelos, Morelos, México

Jslsgrc02@gmail.com

https://orcid.org/0000-0003-3853-6460

\section{Resumen}

El presente estudio se llevó a cabo en la comunidad de Huajintlán, Morelos, México. Su objetivo fue conocer la opinión de los actores de la comunidad sobre las actividades realizadas mediante la vinculación con los estudiantes del Sistema de Integración al Medio Rural (SIMR). Se realizó una investigación de tipo cualitativa con la aplicación del método fenomenológico; la muestra se compuso de 30 personas, hombres y mujeres, cuyas edades oscilaban entre 24 y 65 años. Para la recolección de datos se utilizó un instrumento con 17 ítems, el cual se aplicó a los pobladores de la comunidad que participaron en el desarrollo de las actividades con los alumnos. 


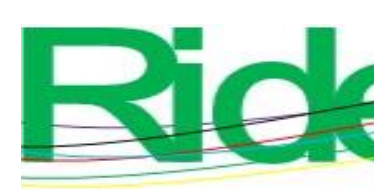

\section{Revista Iberoamericana para la Investigación y el Desarrollo Educativo ISSN $2007-7467$}

Los resultados arrojaron que la primordial forma de inserción hacia la comunidad por parte de los estudiantes del SIMR se dio a través de las acciones realizadas con los niños que cursaban la primaria (84\%). Las principales actividades que llevaron a cabo los participantes de la comunidad con los estudiantes fueron la elaboración de mermeladas, mazapán y nieve (53 \%), las cuales representaron una alternativa para generar ingresos, contribuir al gasto familiar y propiciar un intercambio de saberes entre los educandos y los habitantes de la comunidad; asimismo, manifestaron su disposición para contratar un estudiante una vez que egrese. El binomio vinculación-interacción está presente dentro de las actividades contempladas en la unidad de aprendizaje del SIMR de la Licenciatura en Desarrollo Rural. A través de este proceso se generaron beneficios y necesidades, los cuales son considerados como parte de los métodos que fomentan el crecimiento y desarrollo de los participantes mediante el contraste, la relación y la aplicación de la información recibida en el aula contra lo que está pasando en un escenario real en la comunidad.

Palabras clave: aprendizaje, educación, universidad, vinculación.

\section{Abstract}

The presented study was conducted in the community of Huajintlán, Morelos, Mexico. Its presumed objective was to get to know the opinion of the involved individuals from the local community about the performed activities through linkage with students from the Sistema de Integración al Medio Rural (SIMR). A qualitative investigation was carried out with the application of the phenomenological method; the research sample was integrated by 30 people, male and female, whose ages ranged within 24 and 65 years old. An instrument of 17 items was used for data collection, and it was applied to the participants from the local community who took part in the development of activities along with the higher education students.

The results show that the primary form of insertion into the community for the SIMR students turned out to be actions performed with elementary level students (84\%). The main activities performed by the students with the community participants were the elaboration of jam, mazapán (a traditional peanut powder based candy) and ice cream (53\%), which moreover represented an alternative income venue, contributed to family home expenses and propitiated and exchange of knowledge between the higher education students and the community inhabitants; furthermore, the community participants manifested their willingness to hire a student from the research group once he or she graduated. The linkage-interaction binomial is present within the contemplated activities of the SIMR learning unit from the Rural Development Bachelor Degree. 


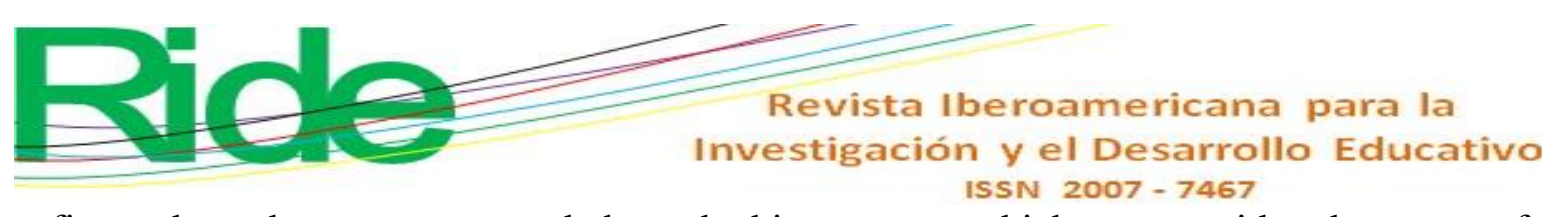

Benefits and needs were generated through this process, which are considered as part of the methods that promote growth and development of the participants by contrasting, linking and applying the knowledge obtained in the classroom in opposition to a real life scenario.

Keywords: knowledge, education, university, linkage.

\section{Resumo}

O presente estudo foi realizado na comunidade de Huajintlán, Morelos, México. Seu objetivo foi conhecer a opinião dos atores da comunidade sobre as atividades realizadas, relacionando-as com os alunos do Sistema de Integração do Ambiente Rural (SIMR). Pesquisa qualitativa foi realizada com a aplicação do método fenomenológico; A amostra foi composta por 30 pessoas, homens e mulheres, cujas idades variaram de 24 a 65 anos. Para a coleta de dados, foi utilizado um instrumento com 17 itens, aplicado aos moradores da comunidade que participaram do desenvolvimento das atividades com os alunos.

Os resultados mostraram que a forma primária de inserção na comunidade pelos alunos do SIMR foi dada por meio das ações realizadas com as crianças que frequentavam a escola primária (84\%). As principais atividades realizadas pelos participantes da comunidade com os alunos foram a elaboração de marmelada, maçapão e neve (53\%), o que representou uma alternativa para gerar renda, contribuir com os gastos familiares e promover uma troca de conhecimentos entre os alunos. alunos e os habitantes da comunidade; Eles também expressaram sua disposição de contratar um aluno quando se formarem. O binômio de interação de links está presente nas atividades contempladas na unidade de aprendizagem SIMR do Curso de Desenvolvimento Rural. Através deste processo, foram gerados benefícios e necessidades, que são considerados como parte dos métodos que estimulam o crescimento e desenvolvimento dos participantes através do contraste, relacionamento e aplicação das informações recebidas em sala de aula em relação ao que está acontecendo. em um cenário real na comunidade.

Palavras-chave: aprendizagem, educação, universidade, vínculo.

Fecha Recepción: Abril 2019

Fecha Aceptación: Agosto 2019 


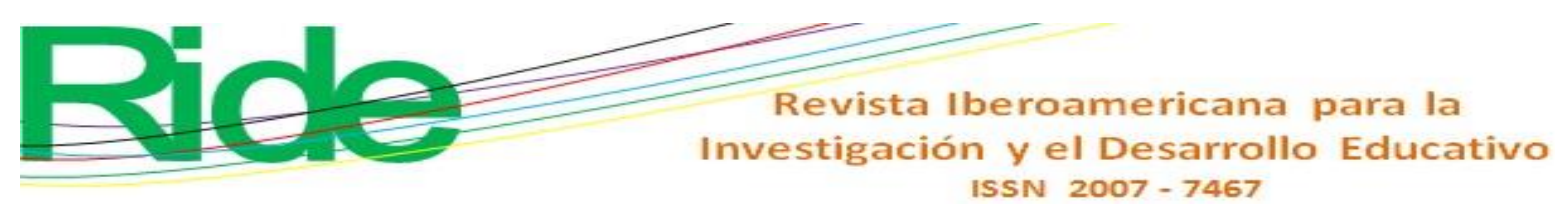

\section{Introducción}

En las universidades se establecen tres funciones sustantivas: la docencia, la investigación y la extensión, las cuales contribuyen al beneficio de los sectores académico, social y productivo. A partir de la Reforma de Córdoba en 1918, la extensión universitaria se centró en tener una relación más estrecha con la sociedad y sus problemáticas, con la intención de generar cambios (Di Meglio y Harispe, 2015); sin embargo, no ha tenido un papel preponderante, no ha sido capaz de privilegiar los procesos vinculatorios entre la universidad y la sociedad, ya que esta permite a las instituciones de educación superior (IES) articular de manera eficiente sus funciones sustantivas al relacionarse con los sectores productivo y social, lo que genera un beneficio mutuo. Es decir, la vinculación "se valida solo en la medida en que se acortan las distancias materiales (físicas), entre la universidad y sociedad" (Saavedra, 2009, p. 106).

Durante varios años las universidades han implementado diversas actividades para lograr una mayor vinculación y cumplir con la interacción; y aunque es una función que no ha tomado fuerza suficiente para participar activa y coordinadamente con los diferentes actores involucrados, es necesario que sea de tipo horizontal, con el propósito de que las investigaciones se realicen de acuerdo con las necesidades de los beneficiarios (sociedad) y estén dentro de las demandas reales (problemática social), binomio que servirá a los estudiantes en el proceso de enseñanza-aprendizaje y a la sociedad misma.

En América Latina, las universidades van quedando cada vez más lejos de los avances científicos y tecnológicos; en el mejor de los casos permanecen difundiendo conocimiento generado en otros rincones del planeta, sin ligar directamente sus acciones al desarrollo nacional o regional y mucho menos en su función social como importante agente en los procesos de consolidación democrática (Gasca y Olvera, 2011, p. 48).

Las universidades en su mayoría llevan a cabo la vinculación a través de la firma de convenios con el sector privado para generar recursos que les permitan fortalecer la infraestructura institucional para el desarrollo de actividades docentes y de investigación. No obstante, aunque esta vinculación ha tenido un impacto favorable, se deberán reforzar las estrategias de inserción comunitaria para el desarrollo de alternativas que contribuyan al mejoramiento de las condiciones de sus pobladores, y cumplir así su finalidad: el fortalecimiento y la transformación de la sociedad (Alvarado, 2009). 


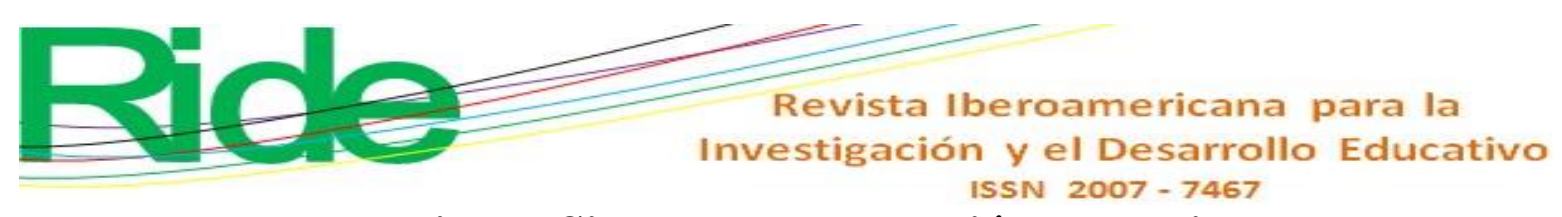

Importancia del Sistema de Integración al Medio Rural

En la Facultad de Ciencias Agropecuarias de la Universidad Autónoma del Estado de Morelos, el currículo de la carrera de Ingeniería en Desarrollo Rural (IDR) estratégicamente cuenta con un SIMR: un modelo educativo de tipo teórico-práctico de formación profesional; un esquema de participación directa y comprometido con los procesos de desarrollo rural que alberga en sí un enfoque interdisciplinario. Su estructura contempla dos grandes fases. La primera, de preparación previa que se aborda a través de los cursos del primero al cuarto semestre. Y la segunda, de integración a la comunidad o escenario de aprendizaje del quinto al octavo (García et al., 2011).

El proceso educativo de participación comunitaria se soporta en la construcción de acciones que de manera simultánea combinan elementos teóricos desde la docencia, considerando las condiciones de la realidad, aplicando el eje metodológico de investigación-acción participativa que permite la aprehensión por el estudiante de la vida comunitaria, desde la cual se derivan investigaciones concretas de carácter técnico productivo, sin perder de vista el eje participativo que conlleva a la apropiación por parte de los actores de la comunidad de transformaciones económicas, sociales, culturales, tecnológicas y ambientales. En este sentido, este sistema contribuye a la inserción del estudiante en escenarios laborales (García et al., 2011).

Los objetivos académicos que plantea este sistema se orientan a que los estudiantes vivencien un liderazgo de promoción del desarrollo comunitario a través de un proceso de integración al medio rural; discernir sobre la visión epistemológica de intervención comunitaria, sobre los métodos y modelos más apropiados de participación comunitaria; aplicar modelos participativos como agente facilitador del desarrollo comunitario; emplear diferentes métodos y técnicas de planeación hacia el desarrollo comunitario; utilizar los conocimientos técnicos de las áreas agrícola, pecuaria y socioeconómica en su proceso de integración desde la etapa de sensibilización hasta la presentación de proyectos de desarrollo comunitario; realizar diagnósticos en áreas de interacción como son: figuras asociativas de las diferentes localidades de los municipios definidos, así como vivenciar en su relación con las comunidades procesos sociales y políticos con la finalidad de sustentar su formación en la esfera afectiva (García et al., 2011).

Mediante la implementación de este modelo de integración comunitaria se pretende propiciar en los estudiantes un aprendizaje significativo, ya que estos, a partir de los conocimientos previos, harán una relación de teoría con el trabajo que desarrollarán en las comunidades. O puesto en los términos de Moreira (2012): 


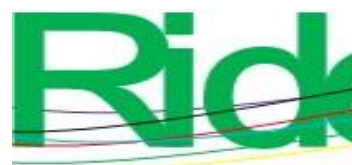

Revista Iberoamericana para la Investigación y el Desarrollo Educativo ISSN 2007 - 7467

[El] aprendizaje significativo es aquel en el que ideas expresadas simbólicamente interactúan de manera sustantiva y no arbitraria con lo que el aprendiz ya sabe. Sustantiva quiere decir no literal, que no es al pie de la letra, y no arbitraria significa que la interacción no se produce con cualquier idea previa, sino con algún conocimiento específicamente relevante ya existente en la estructura cognitiva del sujeto que aprende (p. 30).

Lo anterior indica que es importante considerar los conocimientos previos del alumno, y establecer una relación directa con lo que se requiere que aprenda. Ausubel, Novak y Hanesian (2016) comentan que existen diferentes tipos de aprendizaje: representaciones, conceptos, proposiciones, supraordinario y combinatorio y descubrimientos. En este último "el alumno debe descubrir este contenido por sí mismo, generando proposiciones que representen ya sea soluciones a los problemas que se le planteen o los pasos sucesivos para resolverlo" (p. 64). Sin duda esto se puede lograr a través de la asignatura del SIMR, ya que permite vivenciar aspectos de la realidad.

Como bien se ha mencionado, este sistema favorece la vinculación del estudiante con la realidad social, económica y productiva de las zonas rurales, a fin de desarrollar procesos de retroalimentación a través del aprendizaje vivencial, los cuales, en su mayoría, han sido exitosos. Como lo mencionan Román et al. (2016), los educandos piensan que verdaderamente el trabajo comunitario refuerza los conocimientos teóricos en el área socioeconómica, agrícola y pecuaria. Y García, Alpuche y Yamanaka (2011), por su parte, refieren que los educandos que "participan en SIMR desarrollan actividades de formación basadas en aprendizaje significativo, ya que asocian la teoría con la vida cotidiana y la problemática real, también aprovechan la potencialidad que les muestra el entorno para desarrollar ejercicios interdisciplinarios de formación” (p. 199).

Los estudiantes manifiestan que es necesario participar en las comunidades rurales como IES y no solo como carrera profesional (IDR), porque los habitantes de las comunidades rurales requieren diferentes servicios multidisciplinarios, ya que en ocasiones deben apoyarse de otros profesionistas para resolver algún problema o situación planteada; es decir, es transcendental vincularse internamente (universidad) para trabajar desde un enfoque multidisciplinario y colaborar con los actores involucrados desde una visión holística. Lo anterior coincide con Saavedra (2009), quien señala que la vinculación se concibe "como la relación de la institución en su conjunto con la sociedad...que todas las áreas del conocimiento que cultivan las universidades aporten algo a la sociedad" (p. 107). 


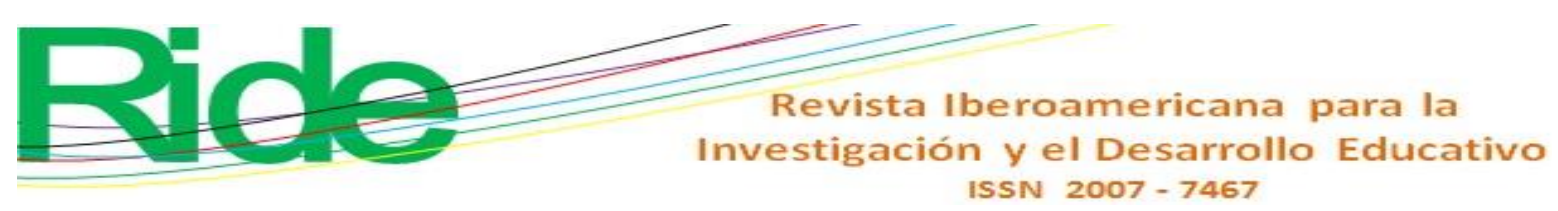

La vinculación universitaria

Vinculación es un término que durante varios lustros se ha considerado como parte de la función sustantiva de extensión de las IES. Sin embargo, tiene diferentes concepciones. Algunos autores la consideran sinónimo de extensión (Mato, 2015); otros afirman que la vinculación no solo debe formar parte de la extensión, sino va más allá: debería ser una función sustantiva más de la universidad (Di Meglio y Harispe, 2015). Pero en lo que sí coinciden es que es una función significativa para el proceso de enseñanza-aprendizaje entre los diversos sectores de la sociedad, es decir, una retroalimentación. Lo que contribuye a la mejora de los actores involucrados porque se generan conocimientos y mejoran habilidades, aptitudes, actitudes y valores. Es una "actividad que indica acción interactiva entre sujetos vivos (los objetos no pueden interactuar sin manipulación), por otra parte, este interactuar se presenta con ligas subjetivas no observables que pueden ser sentimientos, motivos, valores, intereses o correspondencias de persona a persona" (García et al., 2009, p. 108).

La extensión universitaria posee un doble carácter de función y proceso consustancial a la esencia de la actividad universitaria, asumiéndolo como un proceso formativo, dinámico, integrador, multifacético en la comunidad y para la comunidad respaldado por un sistema de formación y capacitación, estimulación, comunicación y aseguramiento que hagan más efectiva dicha labor (Veliz et al., 2011, p. 10).

En otras palabras, la vinculación debe partir de la búsqueda de los talentos sociales como acción interactiva entre los sujetos (García et al., 2009) para insertarse en la realidad (praxis) y conocer las necesidades y problemáticas sociales, económicas y políticas que son pocas satisfechas. Esto a través de acciones coordinadas entre los actores (investigadores-docentes-estudiantessociedad) para apoyar en la disminución o solución de las mismas por medio de las capacidades, habilidades, aptitudes y actitudes de los involucrados (De Aparicio, Chininin y Toledo, 2017).

Numerosas IES ven a la vinculación como una forma de generar financiamiento. Debido a que los proyectos que principalmente se llevan a cabo son con empresas del sector privado, y los que son sociales simplemente dejan de ser importantes o no tienen el mismo realce. Como comenta Saavedra (2009): "La universidad ve a la industria como fuente de recursos financieros y pretende hacer contribuciones intelectuales de importancia para la sociedad y en muchos casos a través de las empresas" (p. 103). 


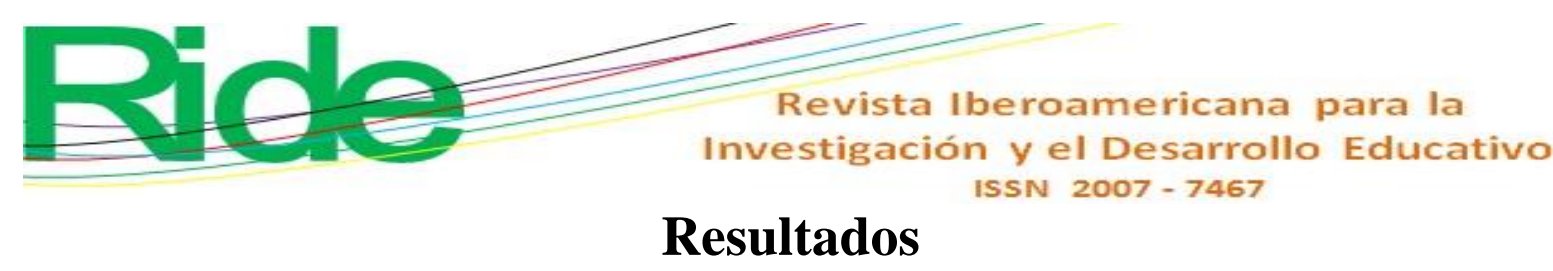

\section{Vinculación SIMR-comunidad rural}

El trabajo de vinculación, como ya se ha mencionado, es un binomio que debe extenderse en las instituciones educativas. Además de ser parte de una función sustantiva, es indispensable para el aprendizaje de los estudiantes, ya que fortalece el desarrollo de sus competencias, habilidades, destrezas y conocimientos adquiridos en el aula. También recupera los saberes tradicionales de los habitantes de la comunidad mediante estos procesos formativos en los cuales todos los actores son beneficiados indistintamente. Como IES existe el compromiso de regresar a la sociedad los conocimientos generados; sin embargo, en la praxis aún es muy escaso el trabajo vinculado con los distintos actores de la sociedad. Por ende, la importancia de la participación de situar a los educandos en la realidad de las comunidades como un proceso previo de preparación para su vida profesional, proceso en el cual se relaciona a estos con sus futuros empleadores y en el que los participantes obtienen conocimiento que impactará en el desarrollo de sus actividades cotidianas.

Por ello, en este trabajo se presentan los resultados obtenidos del instrumento de investigación aplicado a los habitantes de la comunidad de Huajintlán, Morelos, quienes desarrollaron diversas actividades con los estudiantes de la carrera de IDR.

Como parte de estas actividades, los estudiantes se integraron a la comunidad de Huajintlán para desarrollar acciones con los pobladores. El propósito fundamental fue el de fortalecer y reforzar los conocimientos adquiridos en el aula y contribuir en algunas necesidades requeridas por la comunidad, además de implementar sus habilidades para lograr integrarse y, de esta manera, desarrollar el encuadre planteado en la guía pedagógica del propio SIMR, pues "adquirir conocimientos y aplicarlos en beneficio de la sociedad a la que se pertenece proporciona satisfacción, bienestar, plenitud y seguridad" (Pérez, 2019, p. 3).

Ahora bien, a modo de contexto, es necesario indicar que particularmente dos estudiantes del grupo contactaron a un profesor quien hasta hace algunos años se desempeñó como investigador de la Facultad de Ciencias Agropecuarias y quien durante su trabajo comunitario realizó algunas investigaciones en el poblado aquí ya mencionado en varias ocasiones. Dicho investigador, entre otras sugerencias, comentó a los alumnos que en esa localidad se requerían alternativas para impulsar el desarrollo local. Por tal motivo, se expuso ante todo el grupo y a los profesores responsables de coordinar el SIMR la posibilidad de trabajar en la comunidad de Huajintlán, ya que en un principio se contemplaron otras. 


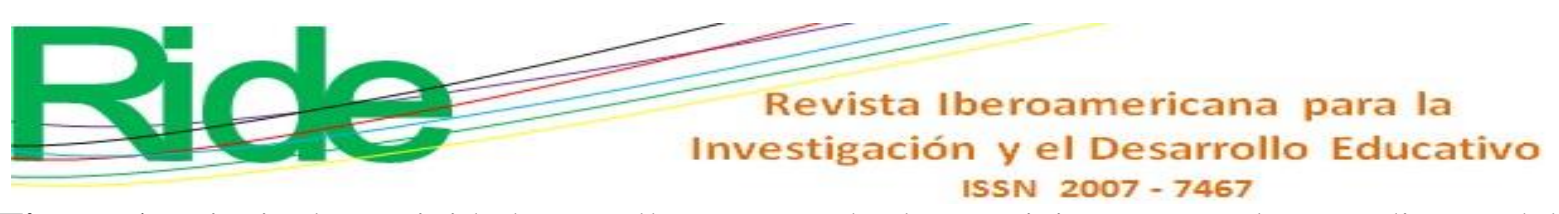

Figura 1. Principales actividades que llevaron a cabo los participantes con los estudiantes del SIMR

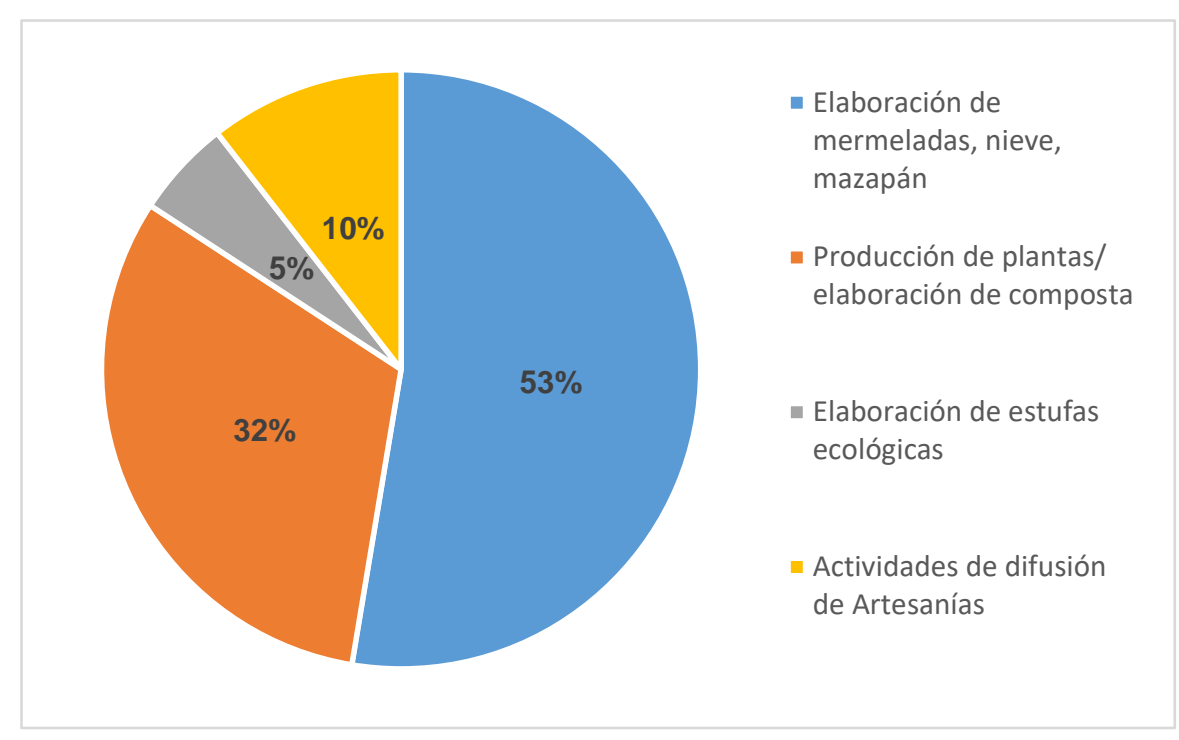

Fuente: Elaboración propia.

Asimismo, es interesante destacar que, a pesar de que los estudiantes concluyen su labor académica y se retiran de la comunidad por terminar los créditos de la materia, la mayoría de los pobladores participantes (79 \%) continúa realizando algunas de las actividades aprendidas e implementadas durante el SIMR, ya sea por ser parte de su alimentación o en apoyo a su economía familiar. Incluso algunos dijeron que la actividad económica aprendida se convirtió en un negocio y venden sus productos en fiestas (ver figura 2). Por ejemplo: una de las participantes compartió que "gracias a los muchachos, ahora me dedico a elaborar y vender nieve en las fiestas de la comunidad, y con eso le ayudo a mi marido con el gasto de la casa" (Cervantes, 13 de abril de 2018, comunicación personal). 


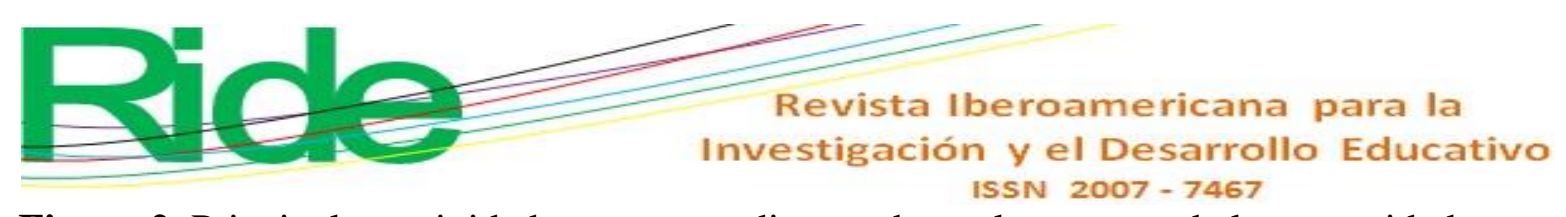

Figura 2. Principales actividades que aprendieron a hacer los actores de la comunidad y que continúan realizando

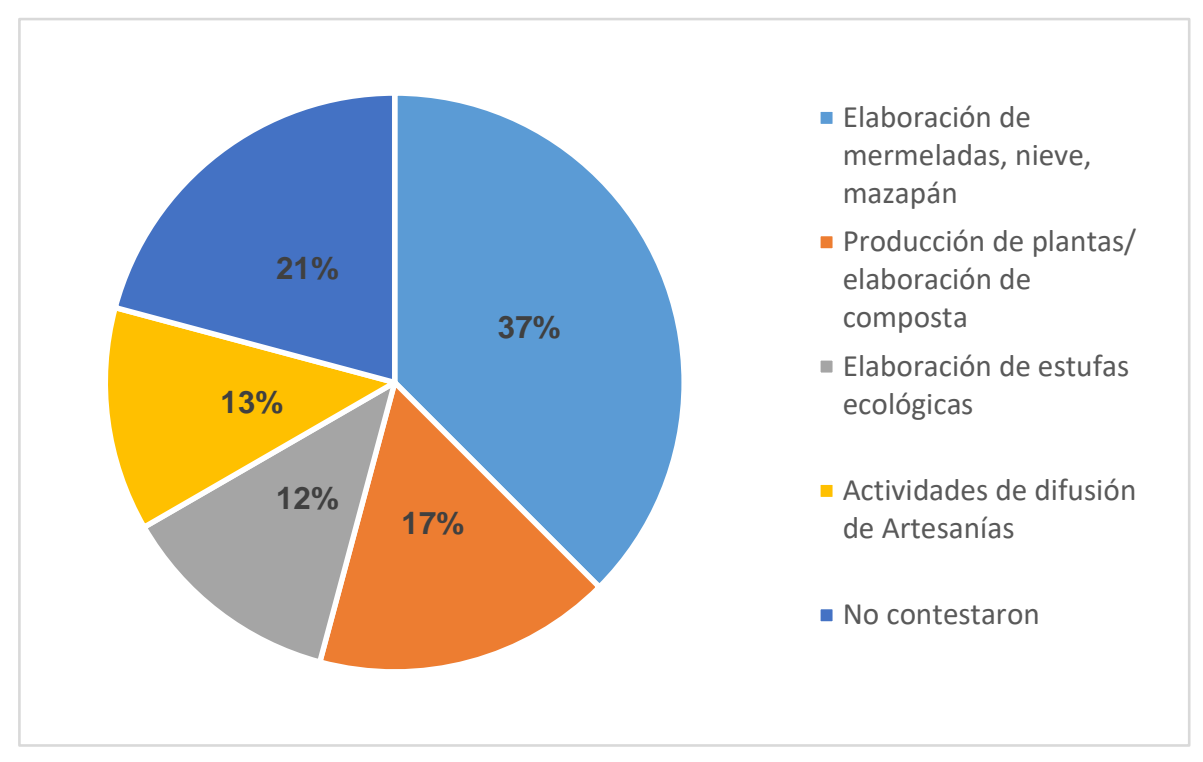

Fuente: Elaboración propia

Las personas beneficiadas con el SIMR en su mayoría fueron mujeres (78 \%). Como ya se ha mencionado, aprendieron e implementaron actividades económicas de transformación y comercialización de productos agropecuarios, entre ellos: elaborar mermelada artesanal, nieve y mazapán. Cabe señalar que en esta comunidad la mayor parte de los pobladores tienen traspatios con árboles frutales y en la temporada de fructificación se desperdicia abundante fruta; por lo tanto, decidieron transformar esta producción y darle valor agregado. Este tipo de procesos motiva a las personas para continuar realizando estas actividades porque obtienen beneficios como: conocimientos (42\%), ahorro de tiempo y dinero (26\%), alimentación sana (11\%) —el porcentaje restante no especificó ningún beneficio (21\%). Al respecto, Eustaquio (5 de abril de 2018, comunicación personal), uno de los habitantes de esta comunidad, comentó lo siguiente:

Yo, por cuestiones de trabajo, no pude asistir a la elaboración de los productos, pero mis niños trabajaron como sus maestros de Cuernavaca [así fueron llamados los estudiantes en la comunidad]; llegaban contentísimos diciéndome: “iMira, lo que hicimos hoy!", describiendo los productos elaborados, como las mermeladas, mazapán y nieve.

Igualmente, los encuestados manifestaron que de las acciones que realizaron con los estudiantes las que más les gustó o benefició fueron las siguientes: elaboración de nieve (26 \%), cuidado de las plantas (16\%), elaboración de mermelada (11\%), mazapán (11\%), composta (5 $\%)$, estufas ecológicas (5\%) y difusión de las artesanías (5\%); el resto no especificó. Con el 


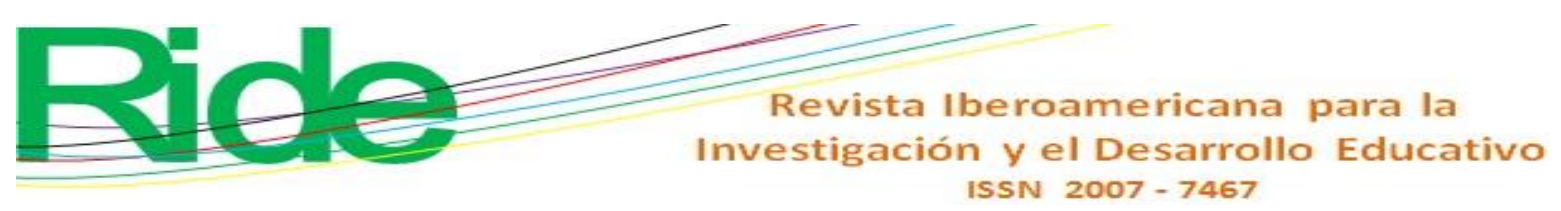

aprendizaje de estas actividades, las personas dicen que pueden seguir practicando lo aprendido para implementarlo en su hogar y/o hacer negocio para contribuir al ingreso de la familia. González (2011) coincide con lo anterior y argumenta lo puesto a continuación:

Se trata de que los saberes salgan de la universidad, se siembren y se adopten en la comunidad para implementar acciones de desarrollo y bienestar social; y al mismo tiempo, que los propios saberes de la comunidad regresen a la universidad para florecer renovados y recreados bajo la rigurosidad de la crítica y la creación de conocimiento (p. 383).

Respecto a la valoración sobre las habilidades, destrezas, actitudes y conocimientos de los estudiantes, la mayor parte (63\%) comentó que tienen conocimientos necesarios y que tienen la capacidad para compartirlos, $21 \%$ dijo que eran buenos, mientras que el resto no contestó. Estas actividades fomentaron un intercambio de saberes que retroalimentaron los conocimientos previos de los actores involucrados, es decir, se llevó a cabo un aprendizaje significativo. Como se observó, la mayoría (95\%) de los participantes consideran que los conocimientos mostrados por los estudiantes son suficientes para proponer alternativas en beneficio de los pobladores de la comunidad. A saber: la generación de nuevos conocimientos, ingresos, reducción de costos, beneficios a la salud y aprovechamiento de los recursos existentes. No obstante, los participantes manifestaron inconformidad porque los estudiantes solo están en la comunidad por un periodo de cuatro semestres. Esto debido a que cada dos años, cuando inicia la asignatura del SIMR, se van eligiendo diferentes lugares en los que llevarán a cabo un nuevo proceso de inserción comunitaria.

El total de los encuestados y entrevistados dijeron que sí están dispuestos a contratar a un estudiante cuando egrese gracias a la experiencia que tuvieron en el SIMR. Incluso plantearon la posibilidad de que los estudiantes continúen el trabajo con las personas que estén dispuestas a contratarlos, o en su caso, gestionar recursos con las autoridades municipales y los beneficiarios para poder cubrir sus honorarios. Por lo tanto, es de resaltar que el trabajo comunitario, cuando se lleva a cabo con responsabilidad, honestidad, compromiso y sobre todo cuando genere algún tipo de beneficio a los involucrados, refuerza la acción vinculatoria para desempeñarse en un ámbito laboral. 


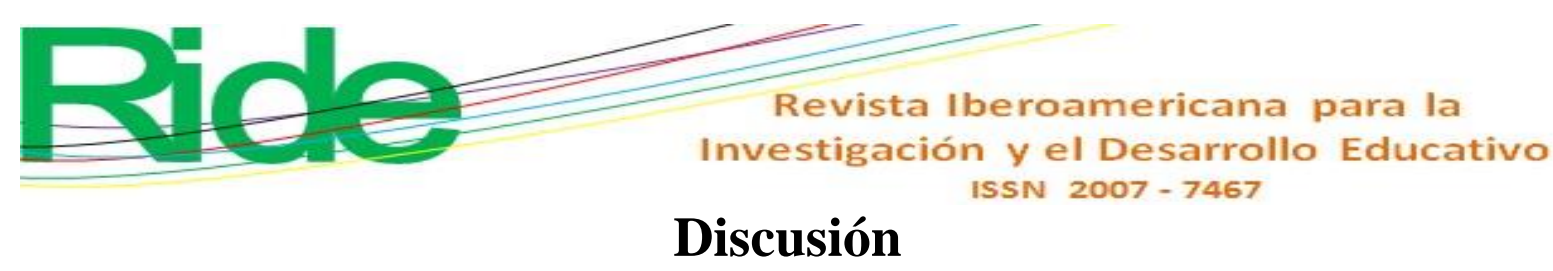

El SIMR se ha llevado a cabo durante algunos años como un proceso de vinculación universitaria e inserción comunitaria. En dicho sistema el estudiante pone a prueba sus conocimientos previos con la finalidad de implementar acciones con los pobladores de las comunidades que permitan adquirir aprendizajes tanto a estos como a aquellos, así como contrastar lo visto en el aula con lo que sucede fuera de esta. Así, se puede conocer la forma en que interactúa el alumno con los pobladores del medio rural. De igual manera, descubrir cómo es que se lleva a cabo ese proceso de contrastar, relacionar y aplicar la información recibida en el aula contra lo que está pasando en un escenario real; qué competencias y capacidades deberá tener el alumno para lograr transmitir y compartir mutuamente saberes con los pobladores de la localidad; qué estrategias y técnicas se requieren para la trasmisión y aseguramiento de la asimilación de la información ofrecida a los pobladores de la comunidad, así como de la apropiación de estos aprendizajes y su permanencia a través del tiempo, si es que se siguen replicando los conocimientos con otros pobladores de la localidad o con sus familiares y contribuyendo en ocasiones a mejorar el ingreso familiar.

Es importante recalcar que si bien se han publicado algunos trabajos que hablan de la importancia de esta vinculación, se enfocan principalmente en los beneficios que adquieren los estudiantes, y existe literatura limitada sobre los estudios relacionados con la percepción de los actores involucrados en estas actividades que desarrollan los educandos como parte de su proceso formativo. Es decir, cuál es la opinión que guardan a este respecto las personas que realizan diversas actividades de vinculación con los estudiantes. Por lo que es trascendental este trabajo de investigación, ya que con los resultados de esta interacción se puede dimensionar cuáles han sido las aportaciones recíprocas hacia la comunidad por permitir a los estudiantes vivenciar procesos comunitarios, a fin de determinar de qué manera están impactando los programas educativos en el desarrollo de competencias y alternativas de desarrollo que los pobladores puedan aplicar con el propósito de contribuir a la generación de conocimientos, y en un momento determinado a la contribución en la generación de recursos económicos.

Por otro lado, la información recopilada se puede utilizar para orientar los diseños y rediseños curriculares con acciones de vinculación en escenarios donde los alumnos relacionen e implementen sus conocimientos previos con situaciones reales y en la solución de problemáticas; escenarios que permitan fortalecer su formación personal y profesional. También esta información será útil para coadyuvar en la orientación de algunas líneas de investigación que tengan un mayor 


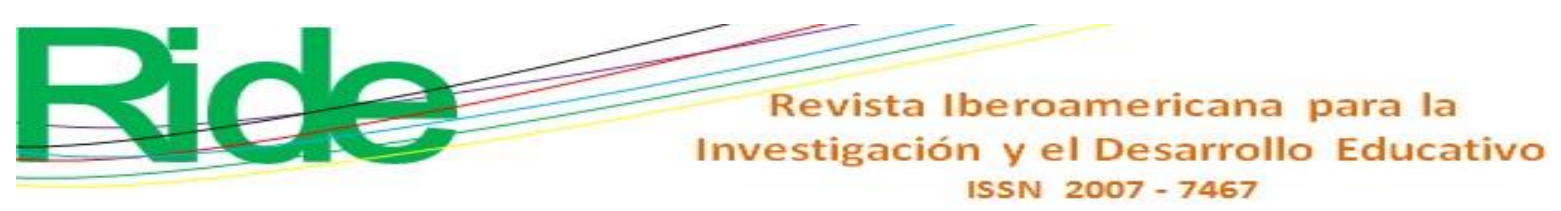

impacto en la solución de problemas y en el planteamiento de estrategias que permitan solventar las necesidades de los pobladores rurales.

\section{Conclusiones}

De acuerdo con las opiniones mencionadas por parte de los encuestados y entrevistados de la comunidad de Huajintlán, se concluye que el desarrollo de actividades por parte de los alumnos del SIMR beneficia a la comunidad porque aprenden a hacer diversas actividades. Entre estas, destacan las siguientes: adquieren conocimiento sobre una alimentación sana, y aprenden sobre la elaboración de nieve, mazapán, mermelada, estufas ecológicas, compostas, entre otras. Asimismo, la mayoría comentó que los estudiantes tienen los conocimientos necesarios y la capacidad para compartirlos. También, consideran que los conocimientos mostrados por los estudiantes son suficientes para proponer alternativas de desarrollo en la comunidad. Por último, promueven el interés de los alumnos de educación primaria por cultivar vegetales y elaborar productos.

Este proceso de desarrollo da lugar a un aprendizaje significativo, ya que se autoconstruye en función de problemas relevantes que se abordan a través de estrategias cognoscitivas, cuyas actividades son de doble vía, lo cual da como resultado un profesional con una visión integral de procesos de desarrollo.

La vinculación, a pesar de formar parte sustantiva de las universidades, continúa sin ser atendida con la importancia debida, sobre todo la que se lleva a cabo con las comunidades rurales, puesto que la mayoría de las IES buscan tener vínculos con empresas privadas, las cuales pueden financiar sus proyectos. No obstante, las universidades están obligadas a regresar a la sociedad los aprendizajes adquiridos. Por ello es importante que los programas académicos contemplen la integración de los estudiantes-docentes-sociedad para desarrollar actitudes, competencias y valores que demanda la profesión. 


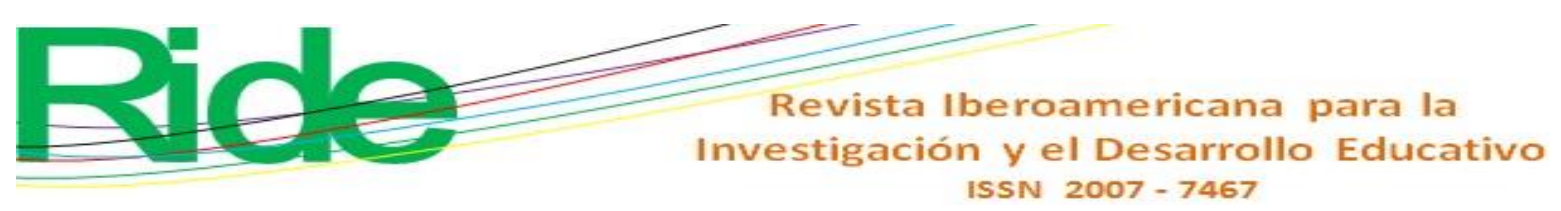

Gasca, E. y Olvera, J. C. (2011). Construir ciudadanía desde las universidades, responsabilidad social universitaria y desafíos ante el siglo XXI. Convergencia Revista de Ciencias Sociales, $\quad$ 18(56), 37-58. $\quad$ Recuperado de http://www.redalyc.org/articulo.oa?id=10516855002.

González, F. (2011). La vinculación universitaria en el modelo de educación superior intercultural en México. La experiencia de un proyecto. Ra Ximhai, 7(3), 381-394. Recuperado de http://www.redalyc.org/pdf/461/46121063007.pdf.

Instituto Nacional de Estadística y Geografía [Inegi]. (2010). Censos y conteos de población y vivienda. México: Instituto Nacional de Estadística y Geografía. Recuperado de www3.inegi.org.mx/sistemas/iter/consulta_info.aspx.

Izcara, S. P. (2014). Manual de Investigación Cualitativa. Ciudad de México, México: Fontamara. Recuperado de https://www.researchgate.net/profile/Simon_Izcara_Palacios/publication/271504124_MA NUAL_DE_INVESTIGACION_CUALITATIVA/links/58949ab192851c54574b9fe7/MA NUAL-DE-INVESTIGACION-CUALITATIVA.pdf.

Kvale, S. (2011). Las entrevistas en la investigación cualitativa. Madrid, España: Morata.

Mato, D. A. (2015). Vinculación social universitaria en Argentina: diversidad de orientaciones de trabajo, logros y dificultades de las experiencias apoyadas por el Programa Nacional de Voluntariado Universitario. CPU-e, 20, 132-149. Recuperado de https://ri.conicet.gov.ar/bitstream/handle/11336/51118/CONICET_Digital_Nro.6e63a3a56bd0-4616-a38f-fcd3f7f604bd_A.pdf?sequence=2\&isAllowed=y.

Mieles, M. D., Tonon, G. y Alvarado, S. V. (2012). Investigación cualitativa: el análisis temático para el tratamiento de la información desde el enfoque de la fenomenología social. Universitas Humanística, 74, 195-225. Recuperado de https://www.redalyc.org/pdf/791/79125420009.pdf

Monje, C. A. (2011). Metodología de la investigación cuantitativa y cualitativa. Guía didáctica. Neiva, Colombia: Facultad de Ciencias Sociales y Humanas, Universidad Surcolombiana. Recuperado de https://www.uv.mx/rmipe/files/2017/02/Guia-didactica-metodologia-de-lainvestigacion.pdf.

Moreira, M. A. (2012). ¿Al final, qué es aprendizaje significativo? QURRICULUM, 25, 29-56. Recuperado de https://riull.ull.es/xmlui/bitstream/handle/915/10652/Q_25_\%282012\%29_02.pdf?sequen ce $=5$ \&isAllowed $=\mathrm{y}$. 


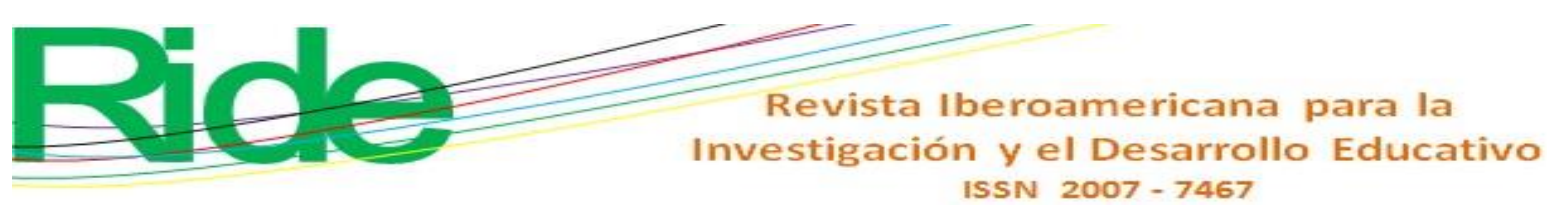

Pérez, L. F. (2019). Consideraciones epistemológicas, psicológicas, sociológicas y pedagógicas de la educación en valores. Iberoamericana para la Investigación y el Desarrollo Educativo, 9(18). Recuperado de https://doi.org/10.23913/ride.v9i18.417.

Román, E., García, F. y Licea, J. E. (2016). La Extensión Universitaria en México y Argentina, dos estudios de caso. ESPAMCIENCIA, 7(2), 167-176. Recuperado de http://190.15.136.171:3050/index.php/Revista_ESPAMCIENCIA/article/view/137/119.

Saavedra, M. L. (2009). Problemática y desafíos actuales de la vinculación universidad empresa: El caso mexicano. Actualidad Contable FACES, 12(19), 100-119. Recuperado de http://www.redalyc.org/html/257/25715409009/.

Trejo, F. (2012). Fenomenología como método de investigación: Una opción para el profesional de enfermería. Enfermería Neurológica (Mex), 11(2), 98-101. Recuperado de https://www.medigraphic.com/pdfs/enfneu/ene-2012/ene122h.pdf.

Véliz, J., Pérez, N., Fernández, Z., Véliz, D. y Pérez N. S. C. (2011). La extensión universitaria y la promoción de salud en la atención primaria, Universidad Médica de Pinar del Río. Ciencias Médicas, 15(4), 218-230. Recuperado de http://scielo.sld.cu/pdf/rpr/v15n4/rpr18411.pdf.

\begin{tabular}{|c|c|}
\hline Rol de Contribución & Autor (es) \\
\hline Conceptualización & Erika Román (principal) Jesús Eduardo Licea (igual) \\
\hline Metodología & Erika Román (principal) Jesús Eduardo Licea (apoyo) \\
\hline Software & No aplica \\
\hline Validación & $\begin{array}{l}\text { Erika Román (principal) Jesús Eduardo Licea (igual) } \\
\text { José Luís Hernández (apoya) }\end{array}$ \\
\hline Análisis Formal & $\begin{array}{l}\text { Erika Román (principal) Jesús Eduardo Licea (igual) } \\
\text { José Luís Hernández (apoya) }\end{array}$ \\
\hline Investigación & Erika Román (principal) Jesús Eduardo Licea (igual) \\
\hline Recursos & Erika Román (igual) Jesús Eduardo Licea (igual) \\
\hline Curación de datos & Erika Román (principal) Jesús Eduardo Licea (igual) \\
\hline Escritura - Preparación del borrador original & Erika Román (principal) \\
\hline
\end{tabular}




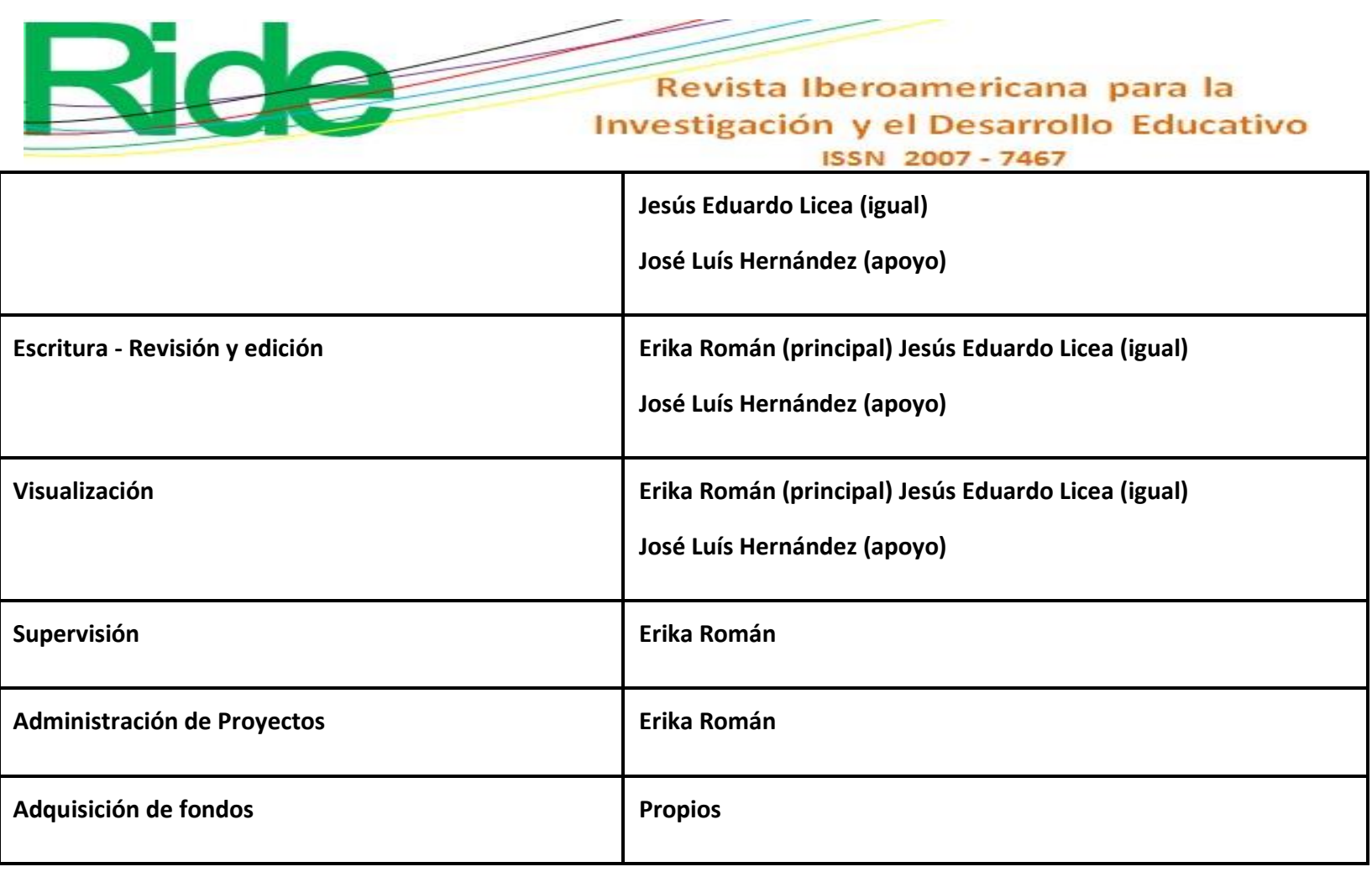

